\title{
Characterization of NIST Human Mitochondrial DNA SRM-2392 and SRM-2392-I Standard Reference Materials by Next Generation Sequencing
}

\author{
Sarah Riman*, Kevin M. Kiesler, Lisa A. Borsuk, Peter M. Vallone \\ U.S. National Institute of Standards and Technology, Biomolecular Measurement Division, \\ 100 Bureau Drive, Gaithersburg, MD 20899-8314, USA \\ *Corresponding author: sarah.riman@nist.gov
}

\begin{abstract}
Standard Reference Materials SRM 2392 and 2392-I are intended to provide quality control when amplifying and sequencing human mitochondrial genome sequences. The National Institute of Standards and Technology (NIST) offers these SRMs to laboratories performing DNA-based forensic human identification, molecular diagnosis of mitochondrial diseases, mutation detection, evolutionary anthropology, and genetic genealogy. The entire mtGenome ( 16569 bp) of SRM 2392 and 2392-I have previously been characterized at NIST by Sanger sequencing. Herein, we used the sensitivity, specificity, and accuracy offered by next generation sequencing (NGS) to: (1) re-sequence the certified values of the SRM 2392 and 2392-I; (2) confirm Sanger data with a high coverage new sequencing technology; (3) detect lower level heteroplasmies (<20\%), and thus (4) support mitochondrial sequencing communities in the adoption of NGS methods. To obtain a consensus sequence for the SRMs as well as identify and control any bias, sequencing was performed using two NGS platforms and data was analyzed using different bioinformatics pipelines. Our results confirm five low level heteroplasmy sites that were not previously observed with Sanger sequencing: three sites in the GM09947A template in SRM 2392 and two sites in the HL-60 template in SRM 2392-I.
\end{abstract}

Keywords: Standard Reference Material (SRM), Next generation sequencing, Mitochondrial DNA, Heteroplasmy 


\section{INTRODUCTION}

Sequence analysis of the mitochondrial (mt) genome has proven valuable for evolutionary anthropology, genetic genealogy, and human identification in cases where forensic biological evidence contains minute, highly degraded, or no genomic DNA [1-8]. Mitochondrial DNA (mtDNA) has also been an informative marker for diagnoses of a number of diseases [9-12] and detection of pathogenic point mutation [13].

The Applied Genetics group at the National Institute of Standards and Technology (NIST) has produced well characterized human mtDNA Standard Reference Materials (SRMs) [14-16]. SRMs are intended to provide quality control for various disciplines performing polymerase chain reaction (PCR) and sequencing of human mtDNA for forensic identification, genetic structure of population, and molecular diagnosis of mitochondrial diseases. The mtDNA SRMs can also be used for quality assurance when assigning sequence confidence to in-house control materials. This assures that the DNA sequencing methods and interpretations being used are conducted correctly [14-16].

The most widely used method of mtDNA typing focuses only on a portion of the $\mathrm{mtGenome.} \mathrm{The} \mathrm{standard} \mathrm{assay} \mathrm{is} \mathrm{based} \mathrm{on} \mathrm{Sanger} \mathrm{sequencing} \mathrm{of} \mathrm{the} \mathrm{hypervariable} \mathrm{regions}$ located in the non-coding displacement loop (D-loop) or control region [17-19]. This region contains a high density of sequence variation; however, under certain circumstances [20], examination of the control region only provides limited information and certain individuals will remain indistinguishable. Sequencing the entire mtGenome provides a higher discrimination power that can allow for further resolution of individuals coming from various populations in addition to those with common HVI/HVII types [21-24]. Although sequencing the entire 16569 bases of human mtGenome can be accomplished with existing Sanger methods [25], the workflow can be labor intensive, expensive, and time consuming.

Recently developed next generation sequencing (NGS) technologies have offered manageable workflow capabilities to mtGenome sequencing, increased the depth of coverage at reduced cost and time, and reliably detected intra-individual mtDNA variation [26-30]. With the introduction of bench top DNA sequencers employing different chemistries and technical approaches [31,32], NGS technology has impacted mtGenome variant discovery and been adopted in several research laboratories [21, 29, 30, 33-35]. 
The entire mtGenome of the SRM 2392 and 2392-I have been characterized at NIST by Sanger type sequencing [14-16]. An update of the SRM 2392 certificate of analysis was released in $1 / 12 / 2009$. The updated certificate corrected a sequencing error in the SRMCHR at position 4929 in the mtGenome. Multiple sequencing experiments confirmed a ' $\mathrm{T}$ ' nucleotide present at position 4929 and not a ' $\mathrm{C}$ ' as inferred by the 2003 certificate. In order to support the adoption of NGS technology in forensics and clinical DNA laboratories, NIST has recently undertaken steps to re-sequence the certified mtDNA SRMs using orthogonal NGS platforms. The enhanced sensitivity of NGS can increase the information content of NIST SRMs, reveal any sequence variation that is undetectable with Sangerbased mtDNA analysis, and increase the confidence in the accuracy of the materials through investigating suspected sources of bias.

Here we present the sequencing results of three mtGenomes obtained from NIST mtDNA SRMs 2392 (CHR and GM09947A) and 2392-I (HL-60). To reliably detect sequence variants arising from the DNA SRM templates and identify any potential bias in a specific NGS chemistry and/or informatics pipelines, samples were prepared using two different library preparation chemistries and were sequenced on two different benchtop NGS platforms, the Ion Torrent Personal Genome Machine (PGM) (Life Technologies, San Francisco, CA) and the Illumina MiSeq (Illumina, San Diego, CA). The results were analyzed with various bioinformatics pipelines and compared to the sequencing data obtained with traditional Sanger typing methodology.

This work focuses on the use of NGS methods to assign high confidence base calls in the SRM 2392 series of materials. The purpose of the material is to provide support for laboratories validating and implementing mtDNA testing using NGS technologies. The intention is not to recommend a sequencing platform, bioinformatics software, or a specific threshold for identification of base variants. 


\section{MATERIALS AND METHODS}

SRM Samples

SRM 2392 became available to the public in December 1999 and is packaged in a single box containing three components: (1) extracted DNA from cell culture line CHR; (2) extracted DNA from cell culture line GM09947A; and (3) cloned HV1 region of the DNA template designated CHR containing a C-stretch which covered base pairs 16133 through 16569 and 1 through 40. CHR is an Epstein-Barr virus-immortalized human white blood cells prepared by the American Type Culture Collection (ATCC; Rockville, MD) [15]. GM09947A cell culture line is a human lymphoid cell line that was transformed with the Epstein-Barr virus and immortalized as a cell culture line by Life Technologies, Inc. (Grand Island, NY) [15]. Both cell culture lines were obtained from normal individuals [15]. SRM 2392-I, first released in 2003, contains one DNA template and is certified for the sequences of the human mtDNA from a promyelocytic cell line (HL-60) derived from the peripheral blood leukocytes from an individual with acute promyelocytic leukemia [14]. The DNA from CHR was extracted using a procedure that enhanced the concentration of mtDNA and reduced, versus fully eliminating, the nuclear DNA. The DNA isolated from GM09947A and HL-60 is the total extracted DNA including nuclear DNA [14-16]. To verify the primary DNA sequence for the mtGenomes of NIST SRM 2392 and 2392-I (NIST, Gaithersburg MD, USA), the materials were sequenced on the Ion Torrent Personal Genome Machine (PGM) (Life Technologies, San Francisco, CA) and Illumina MiSeq (Illumina, San Diego, CA) platforms. SRM 2392 CHR, SRM 2392 GM09947A, and SRM 2392-I will be referred to in the text as SRM-CHR, SRM-9947A, and SRM-HL-60, respectively.

\section{PCR amplification of $m t D N A$}

MtGenomes were first amplified according to the Human mtDNA Genome Guide for the Illumina Sequencing Platform (Part \# 15037958 Rev. B, Illumina) [36]. Two separate PCRs were amplified that produced two long amplicons (9.1 kb and $11.2 \mathrm{~kb}$ ) spanning the entire 16569 bases of the human mtDNA genome [36, 37]. The TaKaRa LA Taq kit (Takara Clontech, Mountain View CA, USA) was used to amplify the mtGenome of the SRM components. Thermal cycling parameters were: initial denaturing for 5 minutes at $94{ }^{\circ} \mathrm{C}$ then 30 cycles of $\left(98^{\circ} \mathrm{C}\right.$ for $15 \mathrm{~s}, 68{ }^{\circ} \mathrm{C}$ for $10 \mathrm{~s}$ [ramp rate $0.2^{\circ} \mathrm{C} / \mathrm{s}$ ], $60{ }^{\circ} \mathrm{C}$ for $15 \mathrm{~s}$, then 68 
${ }^{\circ} \mathrm{C}$ for 11 minutes), followed by a final extension at $72{ }^{\circ} \mathrm{C}$ for 10 minutes and $4{ }^{\circ} \mathrm{C}$ hold. Reaction conditions were: 1 X LA PCR Buffer II (with $25 \mathrm{mM} \mathrm{MgCl}$ ), 400 nM dNTPs, 400 nM each primer, 2.5 Units TaKaRa LA Taq, and 1 ng of genomic template DNA. PCR products were quantified on an Agilent Bioanalyzer 2100 (Agilent, Santa Clara CA, USA) system with the DNA 12,000 Kit. Based on the quantitation results, amplicons were adjusted to a concentration of $0.2 \mathrm{ng} / \mu \mathrm{L}$ with molecular biology grade water, and PCR products for each sample were combined on an equal-volume basis. To assess the reproducibility and precision of the PGM system, two PCR replicates were performed and sequenced for each SRM sample, with each replicate incorporating different barcode sequences.

\section{Library Building and Sequencing on Ion PGM Torrent Platform}

A total of approximately 300 ng of input DNA was used for automated library preparation using the AB Library Builder System (ThermoFisher Scientific), Ion Plus Fragment Library Kit for AB Library Builder System (ThermoFisher Scientific) and IonXpress Barcode Adapters 1-16 Kit (ThermoFisher Scientific) according to the manufacturer's recommended procedure [38]. Size selection was performed off-platform with the Agencourt Ampure XP (Beckman Coulter, Brea CA) system. Size selected library was quantitated using the Ion Library TaqMan Quantitation Kit (ThermoFisher Scientific) on an ABI 7900HT Fast Real-Time PCR System (ThermoFisher Scientific) [39], and libraries were diluted to a concentration of $20 \mathrm{pM}$ for subsequent pooling and sequencing template preparation. Templated Ion Sphere Particles were prepared and loaded into an Ion 318 Chip v2 BC (ThermoFisher Scientific) by the Ion Chef System (ThermoFisher Scientific) using the Ion PGM Hi-Q Chef Kit (ThermoFisher Scientific) [40]. Sequencing was performed on the PGM using 500 flows (200 bp read length).

Initial data analysis was performed with Torrent Server Suite software version 4.4.3 (ThermoFisher Scientific) to generate FASTQ files which were then analyzed for variants relative to the Homo sapiens mitochondrion reference genome (NCBI Reference Sequence NC_012920.1) using different bioinformatic pipelines.

\section{Library Building and Sequencing on Illumina MiSeq Platform}


For Illumina MiSeq sequencing, PCR products were diluted to a nominal concentration of $0.2 \mathrm{ng} / \mu \mathrm{L}$ in molecular biology grade water. Libraries were generated using the Nextera XT DNA Library Preparation Kit with the Nextera XT Index Kit V2 Set A (Illumina) according to the manufacturer's recommended procedure [36]. Libraries were quantitated with the ABI Prism Library Quantitation Kit for Illumina Platforms (Kapa Biosystems, Wilmington MA, USA). Based on the results of the quantitation, libraries were adjusted to a concentration of $20 \mathrm{pM}$ then pooled and sequenced on the MiSeq using a MiSeq Reagent Kit v3 (600 Cycles) (Illumina).

\section{Data Analysis}

Identification of heteroplasmic and homoplasmic sites from the NGS data was analyzed using CLC bio Genomics Workbench version 9.0 (Qiagen) [41], Galaxy [42-45], and GeneMarker® HTS (GM-HTS) (SoftGenetics, Inc.) [46, 47]. Detailed analysis parameters for CLC and Galaxy software pipelines are shown in Figures S1 and S2, respectively.

For the detection of point heteroplasmies in GM-HTS, reads in FASTQ format were aligned to the entire circularized mtDNA revised Cambridge Reference Sequence rCRS [48], using the following alignment options: built in motif file, default values for both identity and soft clipping based on the sequencer used. Variant filter settings were as follows: variant percentage $\geq(20 \%, 10 \%, 5 \%$, and $1 \%)$, variant allele coverage $\geq 40$, total coverage $\geq 200$, allele score difference $\leq 10$, and allele balance ratio for SNPs $(\leq 2.5)$ and indels $(\leq 5.0)$.

\section{Heteroplasmy Verification using STRait Razor}

To further verify the accuracy of detection of heteroplasmy calls obtained from CLC Bio, Galaxy and GM-HTS, FASTQ files generated with the PGM and MiSeq were directly analyzed with the STR Allele Identification Tool - Razor (STRait Razor) [49, 50]. Sequence reads containing both leading and trailing flanking regions (Table S1) directly adjacent to the identified length and point heteroplasmy were extracted from the NGS-based mtDNA SRM raw data by modifying the STRait Razor configuration file with mtDNA recognition sequences flanking the regions of interest. The filtered reads were evaluated and counted. 
The frequency of heteroplasmy was calculated by dividing the number of filtered reads containing the variant by the total number of filtered reads.

\section{RESULTS AND DISCUSSION}

MtGenome Depth of Coverage for the Three SRM Samples Across the PGM and MiSeq Platforms

As has been previously observed [51-53], mitochondrial sequencing results in a varying range of depth of coverage depending on the platform, degree of multiplexing, and alignment method used. As observed from reads aligned in CLC software, the average depth of sequencing coverage on the MiSeq for SRM-CHR, SRM-9947A, and SRM-HL-60 was 16k (range 3,758-41,065), 17k (range 3,088-46,408), and 21k (range 3,504-55,548), respectively. Samples sequenced on the PGM had a lower average depth of coverage than those sequenced on the MiSeq. For example, samples sequenced on the PGM and aligned in CLC yielded an average read depth of 4k, range of: 732-10,941 for SRM-CHR, 791-10,667 for SRM-9947A, and 755-10,581 for SRM-HL-60. This coverage variation is attributed to the higher total sequence output of the MiSeq platform as compared to the Ion Torrent PGM [51].

\section{mtDNA SRM Homoplasmic Variants}

Variants, positions across the mtGenome where the primary base differs from the reference rCRS, were analyzed for each of the SRM mtDNA sequences analyzed. Table 1 shows all the variants found in SRM-CHR, SRM-9947A, and SRM-HL-60 mtDNA sequences generated on both the MiSeq and PGM platforms. Also, Table 1 shows that the majority of the variants are homoplasmic (>98\%), previously characterized with the Sanger Sequencing method, and reported in the published certified reference materials for the mtDNA SRMs [14-16].

We detected 33, 17, and 32 homoplasmic variants in SRM-CHR, SRM-9947A, and SRM-HL-60 NGS data, respectively. The consensus data from the different NGS platforms for the mtDNA SRMs was in concordance with certified values determined by the Sanger Sequencing typing method for all homoplasmic variants. 


\section{Sequence Heteroplasmy Discovery in the NIST Human mtDNA SRM-9947A and SRM-} HL-60

Heteroplasmy is the co-existence of multiple types of mtDNA sequences within the same cell, tissue, or individual [54, 55]. Heteroplasmy can be observed as: point heteroplasmy (PHP) and/or length heteroplasmy (LHP). PHP is the presence of mixture of two DNA bases at a single position. LHP is the presence of at least two populations of mtDNA sequences that differ in the number of nucleotides and thus their length [55-58]. LHP occurs in the C-stretches of HV I (16184-16193), II (303-315), and III (568-573) regions as well as in the AC repeat region (515-524) of the HVIII [58-60]. Both types of heteroplasmic variation have been previously observed within mtDNA SRMs by Sanger sequencing [14-16].

Due to the higher sensitivity and coverage at each mtDNA site, an increase in the number of heteroplasmy positions were further detected using the NGS technologies. To have confidence in the heteroplasmy call, only variants which were observed across all NGS sequencing experiments on different platforms, with the different bioinformatics pipelines, and further confirmed with STRait-razor method $[49,50]$ were considered to be novel heteroplasmic sites.

Previously with Sanger sequencing, one instance of PHP was observed in SRM-CHR at position 64 (C/T) (herein Reference/Variant). When SRM-CHR was sequenced on the NGS platforms, no further heteroplasmy sites were detected across the platforms, PCR replicates, and pipeline analyses (Table 1A).

Three new PHP sites were identified from the PGM and MiSeq data of SRM-9947A. Table 1B depicts the newly characterized positions at 1393 (G/A), 3242 (G/A), and 7861 (T/C). A previous study also detected the 1393 and 7861 sites from the SRM-9947A mtDNA in data generated on the Roche 454 GS Junior sequencer [34]. The authors validated sites 1393 and 7861 using single base extension assays with both capillary electrophoresis and MALDI-TOF. Also, an early study published by the Applied Genetics Group at NIST on characterizing NIST standard reference materials by NGS also detected both 1393 and 7861 across different NGS platforms including HiSeq 2000 (Illumina) as well as SOLiD 5500 system [61]. 
Table 1C shows two novel PHP sites observed in SRM-HL-60: 2445 (T/C) and 5149 (C/T). The PHP at 12071 (T/C) was characterized previously [14]. Five novel heteroplasmies discovered in the human mtDNA SRM-9947A and SRM-

248 HL-60 were not reliably detected by the Sanger sequencing studies performed at NIST and 249 thus were not reported in the NIST SRM 2392 and 2392-I certificates. However, 64 (C/T) in 250 SRM-CHR and 12071 (T/C) in SRM-HL-60 were further confirmed and quantitatively 251 evaluated with this NGS study. Together these results show that the NGS system is 252 extremely sensitive, increases the amount of information from the mtDNA SRMs, and 253 allows detection of rare variants in comparison to Sanger sequencing. 
A.

\begin{tabular}{|c|c|c|c|c|}
\hline $\begin{array}{l}\text { Nucleotide } \\
\text { Position }\end{array}$ & \begin{tabular}{c|} 
rCRS \\
Reference
\end{tabular} & SRM-CHR Sanger Call & $\begin{array}{l}\text { SRM-CHR PGM } \\
\text { Consensus }\end{array}$ & $\begin{array}{c}\text { SRM-CHR MiSeq } \\
\text { Consensus }\end{array}$ \\
\hline 64 & \begin{tabular}{c|}
$\mathrm{C}$ \\
\end{tabular} & $\mathrm{C} / \mathrm{T}$ & $\mathrm{C} / \mathrm{T}$ & $\mathrm{C} / \mathrm{T}$ \\
\hline 73 & A & $\mathrm{G}$ & $\mathrm{G}$ & $\mathrm{G}$ \\
\hline 195 & $\mathrm{~T}$ & $\mathrm{C}$ & $\mathrm{C}$ & $\mathrm{C}$ \\
\hline 204 & $\mathrm{~T}$ & $\mathrm{C}$ & $\mathrm{C}$ & $\mathrm{C}$ \\
\hline 207 & G & A & A & A \\
\hline 263 & A & G & G & G \\
\hline 309.1 & & $\underline{C}$ & & \\
\hline$\underline{315.1}$ & & $\underline{C}$ & & \\
\hline 709 & G & A & A & A \\
\hline 750 & A & G & G & G \\
\hline 1438 & $\mathrm{~A}$ & G & G & G \\
\hline 1719 & G & $\mathrm{A}$ & $\mathrm{A}$ & $\mathrm{A}$ \\
\hline 2706 & $\mathrm{~A}$ & G & $\mathrm{G}$ & $G$ \\
\hline 4769 & A & G & G & G \\
\hline 4929 & $\mathrm{C}$ & $\mathrm{T}$ & $\mathrm{T}$ & $\mathrm{T}$ \\
\hline 5186 & $\mathrm{~A}$ & G & G & G \\
\hline 6221 & $\mathrm{~T}$ & $\mathrm{C}$ & $\mathrm{C}$ & $\mathrm{C}$ \\
\hline 6371 & $\mathrm{C}$ & $T$ & $\mathrm{~T}$ & $\mathrm{~T}$ \\
\hline 6791 & A & G & $\bar{G}$ & $\bar{G}$ \\
\hline 7028 & $\mathrm{C}$ & $\mathrm{T}$ & $T$ & $T$ \\
\hline 8503 & $\mathrm{~T}$ & $\mathrm{C}$ & $\mathrm{C}$ & $\mathrm{C}$ \\
\hline 8860 & $\bar{A}$ & $G$ & G & G \\
\hline 11719 & G & A & A & $\mathrm{A}$ \\
\hline 11878 & $T$ & $\mathrm{C}$ & $\mathrm{C}$ & $\mathrm{C}$ \\
\hline 12612 & $\mathrm{~A}$ & G & $\mathrm{G}$ & $\mathrm{G}$ \\
\hline 12705 & $\mathrm{C}$ & $\mathrm{T}$ & $\mathrm{T}$ & $\mathrm{T}$ \\
\hline 13708 & G & $\mathrm{A}$ & $\mathrm{A}$ & $\mathrm{A}$ \\
\hline 13966 & $\mathrm{~A}$ & G & G & $G$ \\
\hline 14470 & $T$ & $\mathrm{C}$ & $\mathrm{C}$ & $\mathrm{C}$ \\
\hline 14766 & $\mathrm{C}$ & $\mathrm{T}$ & $\mathrm{T}$ & $\mathrm{T}$ \\
\hline 15326 & $\mathrm{~A}$ & G & $G$ & $G$ \\
\hline 16183 & $\mathrm{~A}$ & $\mathrm{C}$ & $\mathrm{C}$ & $\mathrm{C}$ \\
\hline 16189 & $\mathrm{~T}$ & C & $\mathrm{C}$ & $\mathrm{C}$ \\
\hline$\underline{16193.1}$ & & $\underline{C}$ & & \\
\hline 16223 & $\mathrm{C}$ & $\mathrm{T}$ & $\mathrm{T}$ & $\mathrm{T}$ \\
\hline 16278 & $\mathrm{C}$ & $\mathrm{T}$ & $\mathrm{T}$ & $\mathrm{T}$ \\
\hline 16519 & $\mathrm{~T}$ & $\mathrm{C}$ & $\mathrm{C}$ & $\mathrm{C}$ \\
\hline
\end{tabular}


B.

\begin{tabular}{|c|c|c|c|c|}
\hline $\begin{array}{l}\text { Nucleotide } \\
\text { Position }\end{array}$ & $\begin{array}{c}\text { rCRS } \\
\text { Reference } \\
\text { Sequence }\end{array}$ & $\begin{array}{c}\text { SRM-9947A } \\
\text { Sanger Call }\end{array}$ & $\begin{array}{c}\text { SRM-9947A } \\
\text { PGM Consensus }\end{array}$ & $\begin{array}{c}\text { SRM-9947A } \\
\text { MiSeq Cons ensus }\end{array}$ \\
\hline 93 & A & $\mathrm{G}$ & $G$ & G \\
\hline 195 & $\mathrm{~T}$ & $\mathrm{C}$ & $\mathrm{C}$ & $\mathrm{C}$ \\
\hline 214 & A & G & G & G \\
\hline 263 & $\mathrm{~A}$ & G & G & G \\
\hline 309.1 & & $\underline{C}$ & & \\
\hline$\underline{309.2}$ & & $\underline{C}$ & & \\
\hline 315.1 & & $\underline{C}$ & & \\
\hline 750 & $\mathrm{~A}$ & $\mathrm{G}$ & G & G \\
\hline 1393 & G & $\mathrm{G}$ & $G / A$ & $G / A$ \\
\hline 1438 & A & G & $G$ & G \\
\hline 3242 & G & G & G/A & $G / A$ \\
\hline 4135 & $\mathrm{~T}$ & $\mathrm{C}$ & $\mathrm{C}$ & $\mathrm{C}$ \\
\hline 4769 & A & $G$ & G & G \\
\hline 7645 & $\mathrm{~T}$ & $\mathrm{C}$ & C & $\mathrm{C}$ \\
\hline 7861 & $\mathrm{~T}$ & $\mathrm{C}$ & $\mathrm{T} / \mathrm{C}$ & $\mathrm{T} / \mathrm{C}$ \\
\hline 8448 & $\mathrm{~T}$ & $\mathrm{C}$ & $\mathrm{C}$ & $\mathrm{C}$ \\
\hline 8860 & $\mathrm{~A}$ & $G$ & G & G \\
\hline 9315 & $\mathrm{~T}$ & $\mathrm{C}$ & $\mathrm{C}$ & $\mathrm{C}$ \\
\hline 13572 & $\mathrm{~T}$ & $\mathrm{C}$ & $\mathrm{C}$ & $\mathrm{C}$ \\
\hline 13759 & $G$ & A & A & A \\
\hline 15326 & A & G & G & G \\
\hline 16311 & $\mathrm{~T}$ & $\mathrm{C}$ & $\mathrm{C}$ & C \\
\hline 16519 & $\mathrm{~T}$ & $\mathrm{C}$ & $\mathrm{C}$ & C \\
\hline
\end{tabular}


C.

\begin{tabular}{|c|c|c|c|c|}
\hline $\begin{array}{l}\text { Nucleotide } \\
\text { Position }\end{array}$ & \begin{tabular}{|c|} 
rCRS Reference \\
Sequence
\end{tabular} & $\begin{array}{l}\text { SRM-HL-60 } \\
\text { Sanger Call }\end{array}$ & \begin{tabular}{|c|} 
SRM-HL-60 \\
PGM Consensus
\end{tabular} & $\begin{array}{c}\text { SRM-HL-60 MiSeq } \\
\text { Consensus }\end{array}$ \\
\hline 73 & A & G & G & G \\
\hline 150 & $\mathrm{C}$ & $\mathrm{T}$ & $\mathrm{T}$ & $\mathrm{T}$ \\
\hline 152 & $\mathrm{~T}$ & $\mathrm{C}$ & C & C \\
\hline 263 & $\bar{A}$ & G & $\bar{G}$ & $\bar{G}$ \\
\hline 295 & C & $T$ & $\mathrm{~T}$ & $\mathrm{~T}$ \\
\hline$\underline{315.1}$ & & $\underline{C}$ & & \\
\hline 489 & $\mathrm{~T}$ & C & C & C \\
\hline 750 & $\overline{\mathrm{A}}$ & G & $\bar{G}$ & $\bar{G}$ \\
\hline 1438 & $\bar{A}$ & G & G & G \\
\hline 2445 & $\mathrm{~T}$ & $\mathrm{~T}$ & $T / C$ & $\mathrm{~T} / \mathrm{C}$ \\
\hline 2706 & $\bar{A}$ & G & $\mathrm{G}$ & G \\
\hline 4216 & $\mathrm{~T}$ & $\mathrm{C}$ & C & C \\
\hline 4769 & $\mathrm{~A}$ & $G$ & $\mathrm{G}$ & $\mathrm{G}$ \\
\hline 5149 & C & $\mathrm{C}$ & $\mathrm{C} / \mathrm{T}$ & $\mathrm{C} / \mathrm{T}$ \\
\hline 5228 & $\mathrm{C}$ & $G$ & $\mathrm{G}$ & $\mathrm{G}$ \\
\hline 5633 & $\mathrm{C}$ & $T$ & $T$ & $T$ \\
\hline 7028 & $\mathrm{C}$ & $\mathrm{T}$ & $\mathrm{T}$ & $\mathrm{T}$ \\
\hline 7476 & C & $\mathrm{T}$ & $\mathrm{T}$ & $\mathrm{T}$ \\
\hline 8860 & $\mathrm{~A}$ & $G$ & $G$ & G \\
\hline 10172 & $G$ & A & $\bar{A}$ & A \\
\hline 10398 & $\bar{A}$ & G & G & G \\
\hline 11251 & $\mathrm{~A}$ & G & G & G \\
\hline 11719 & G & A & $\bar{A}$ & A \\
\hline 12071 & $\mathrm{~T}$ & $\mathrm{~T} / \mathrm{C}$ & $\mathrm{T} / \mathrm{C}$ & $\mathrm{T} / \mathrm{C}$ \\
\hline 12612 & $\mathrm{~A}$ & $\mathrm{G}$ & G & $\mathrm{G}$ \\
\hline 13708 & $\bar{G}$ & $\mathrm{~A}$ & $\bar{A}$ & $\mathrm{~A}$ \\
\hline 14569 & G & A & $\mathrm{A}$ & $\mathrm{A}$ \\
\hline 14766 & C & $\bar{T}$ & $\bar{T}$ & $\bar{T}$ \\
\hline 15257 & G & $\mathrm{A}$ & $\mathrm{A}$ & $\mathrm{A}$ \\
\hline 15326 & $\mathrm{~A}$ & G & $G$ & G \\
\hline 15452 & $\mathrm{C}$ & $\mathrm{A}$ & $\bar{A}$ & $\mathrm{~A}$ \\
\hline 15812 & $G$ & $\mathrm{~A}$ & $\mathrm{~A}$ & $\mathrm{~A}$ \\
\hline 16069 & C & $\mathrm{T}$ & $\mathrm{T}$ & $\mathrm{T}$ \\
\hline 16193 & $\mathrm{C}$ & $T$ & $\mathrm{~T}$ & $T$ \\
\hline 16278 & $\mathrm{C}$ & $\mathrm{T}$ & $\mathrm{T}$ & $\mathrm{T}$ \\
\hline 16362 & $\mathrm{~T}$ & C & C & C \\
\hline
\end{tabular}

Table 1. Variants reported as differences relative to the rCRS.

255 A. SRM-CHR; B. SRM-9947A; and C. SRM-HL-60. Novel heteroplasmic sites identified by 256 NGS (in bold and highlighted in grey) and that have not been reliably detected using Sanger 257 sequencing are called in the following manner (Nucleotide position where mixed bases are 258 seen followed by the Reference Base/Variant Base). Those sites were: 1393 G/A; 3242 G/A; 259 and $7861 \mathrm{~T} / \mathrm{C}$ in SRM-9947A, and $2445 \mathrm{~T} / \mathrm{C}$ and $5149 \mathrm{C} / \mathrm{T}$ in SRM-HL-60. Heteroplasmy 260 sites previously published in the SRMs certificates, 64 (C/T) in SRM-CHR and 12,071 (T/C) 261 in SRM-HL-60, are highlighted in bold font. Insertions of C residues in the HV1 and HV2 262 regions are underlined and in italics. 


\section{Different Threshold Cutoff Application}

Several pilot analyses were conducted in order to identify the most effective minimum variant frequency setting for use in analysis with CLC bio, Galaxy, and GM-HTS. Software was configured with cutoffs at $20 \%, 10 \%, 5 \%$, and $1 \%$. The observed heteroplasmy sites were initially detected at different cutoff levels (Table 2). When a $20 \%$ variant allele frequency cut-off was applied to CLC, Galaxy, and GM-HTS workflows to analyze the mtDNA SRM data generated on both the PGM and MiSeq, several heteroplasmy sites were called: 64 (C/T) in SRM-CHR, 7861 (T/C) in SRM-9947A, and 12071 (T/C) in SRM-HL-60 (Table 2). As mentioned previously, only 64 (C/T) and 12071 (T/C) had been detected in Sanger sequencing studies with heteroplasmy detection thresholds roughly greater than or equal to $10 \%$. When the threshold was set to $10 \%, 1393$ (G/A) was called in SRM-9947A. Upon applying a 5 \% threshold, 2445 (T/C) and 5149 (C/T) were called in the SRM-HL-60.

A substantial number of calls were reported as variants in the SRM sequences when a $1 \%$ point heteroplasmy filter was applied. At this low cutoff level, the distinction between true mtDNA variants and noise was challenging. Most of the variant calls observed in the MiSeq data were not concordant with those generated on PGM and vice versa (data not shown). However, of all the calls at this very low cutoff level, one particular position of interest 3242 site in SRM-9947A shows what appears to be a low-level heteroplasmy (G/A) and was reproducible across platforms, among PCR replicates, and with the different bioinformatics pipelines. The 3242 was not detected with Sanger studies nor reported in the certificate as a variant. 


\begin{tabular}{|c|c|c|c|}
\hline Threshold & SRM-CHR & SRM-9947A & SRM-HL-60 \\
\hline \multirow{2}{*}{$20 \%$} & $64 \mathrm{C} / \mathrm{T}$ & $7861 \mathrm{~T} / \mathrm{C}$ & $12071 \mathrm{~T} / \mathrm{C}$ \\
\hline \multirow{2}{*}{$10 \%$} & \multirow{2}{*}{$64 \mathrm{C} / \mathrm{T}$} & $1393 \mathrm{G} / \mathrm{A}$ & $12071 \mathrm{~T} / \mathrm{C}$ \\
& & $7861 \mathrm{~T} / \mathrm{C}$ & \\
\hline \multirow{2}{*}{$5 \%$} & \multirow{2}{*}{$64 \mathrm{C} / \mathrm{T}$} & & $2445 \mathrm{~T} / \mathrm{C}$ \\
& & $1393 \mathrm{G} / \mathrm{A}$ & $5149 \mathrm{C} / \mathrm{T}$ \\
& & $7861 \mathrm{~T} / \mathrm{C}$ & $12071 \mathrm{~T} / \mathrm{C}$ \\
\hline \multirow{2}{*}{$1 \%$} & \multirow{2}{*}{$64 \mathrm{C} / \mathrm{T}$} & $1393 \mathrm{G} / \mathrm{A}$ & $2445 \mathrm{~T} / \mathrm{C}$ \\
& & $3242 \mathrm{G} / \mathrm{A}$ & $5149 \mathrm{C} / \mathrm{T}$ \\
& & $7861 \mathrm{~T} / \mathrm{C}$ & $12071 \mathrm{~T} / \mathrm{C}$ \\
\hline
\end{tabular}

Table 2. Detection of PHP sites in each of the mtDNA SRM material as a function of allele calling thresholds.

To be included in the table, PHP sites must be detected on both the MiSeq and PGM platforms, in both PCR replicates, and across the different bioinformatics pipelines. The $64 \mathrm{C} / \mathrm{T}$ and $12071 \mathrm{~T} / \mathrm{C}$ are listed in the current SRM certificates and were previously detected by Sanger sequencing.

\section{Frequency of Heteroplasmy Sites}

We calculated the frequency of heteroplasmic sites by each method to further characterize the reference materials. Frequency was calculated as the number of reads containing the base call divided by the total number of reads covering that position. Quantitative values for the heteroplasmic sites are presented in Table 3.

There was agreement across all NGS platforms and bioinformatics pipelines including STRait Razor as to the identification and quantitation of mixed bases at each of the studied heteroplasmic positions, with the exception of 7861 site in SRM-9947A (Table 3B). We observed variation in the frequency of this specific heteroplasmy with the reads generated on the PGM platform. MiSeq reads contained variant allele $C$ in proportion of $88 \%$ of total reads covering that position and a lower frequency when the same region was sequenced on the PGM platform (Table 3B). As seen in the Integrative Genomics Viewer (IGV) [62], the heteroplasmy site 7861 is located in a region surrounding a stretch of three contiguous $C$ residues at positions 7862 through 7864 (Figure 1). Many PGM reads were interpreted by the software as a false deletion at position 7861. The terminator-free sequencing chemistry utilized by Ion Torrent is known to have difficulty assigning integer 
302 base call values around homopolymer stretches ( $>3$ bases), a situation that applies to 7861 303 [63, 64]. Only PGM reads with mapping and base quality $\geq 20$ were retained by the 304 bioinformatics pipelines (CLC and Galaxy) to compute the variant allele frequency (" $\mathrm{C}$ ") at 305 7861. This filtering step of low quality reads caused a decrease in the average read 306 coverage per 7861 site, and thus a lower frequency level of "C". The pattern of 307 heteroplasmy at 7861 was also evaluated in GM-HTS. PGM reads contained proportions of $308 \sim 83 \%$ variant allele $\mathrm{C}, 13 \%$ of allele $\mathrm{T}$, and $4 \%$ deletion of total reads covering that 309 position. CLC and Galaxy analyses generated a lower variant "C" frequency of $68.5 \%$ and $31078.5 \%$, respectively. Therefore, the variation in frequency calling of this site is attributed to 311 chemistry related limitations as well as different parameter settings in each software (e.g. 312 Q-score filtering, C-stretch resolution).

313 The algorithm of STRait Razor does not take into consideration the quality of bases 314 sequenced. The frequency of all the heteroplasmy sites sequenced on PGM and MiSeq and 315 computed from Strait Razor output was in concordance with the frequencies calculated by 316 CLC, Galaxy, and GM-HTS except for the 7861 site (Table 3). Although the allele frequency 317 at 7861 site computed by STRait Razor was similar to that of MiSeq ( $~ 88 \%$ ), it should be 318 noted that manual inspection of the FASTQ files of the PGM reads containing 7861 319 confirmed lower base quality reads as compared to those sequenced on MiSeq. 


\begin{tabular}{|c|c|c|}
\hline \multirow{2}{*}{ A } & \multicolumn{2}{|c|}{ SRM-CHR } \\
\cline { 2 - 3 } & \multicolumn{2}{|c|}{ Position 64 } \\
\hline Platforms \& Analysis & REF C\% & VAR T\% \\
\hline PGM (CLC) & 69 & 31 \\
\hline MiSeq (CLC) & 68 & 32 \\
\hline PGM (Galaxy) & 67.5 & 32.5 \\
\hline MiSeq (Galaxy) & 69 & 31 \\
\hline PGM (GM-HTS) & 70 & 30 \\
\hline MiSeq (GM-HTS) & 70 & 30 \\
\hline PGM (STRait Razor) & 67 & 33 \\
\hline MiSeq (STRait Razor) & 69 & 31 \\
\hline
\end{tabular}

\begin{tabular}{|c|c|c|c|c|c|c|}
\hline \multirow{2}{*}{ B } & \multicolumn{6}{|c|}{ SRM-9947 A } \\
\hline & \multicolumn{2}{|c|}{ Position 1393} & \multicolumn{2}{|c|}{ Position 3242} & \multicolumn{2}{|c|}{ Position 7861} \\
\hline Platforms \& Analysis & REF G\% & VAR A\% & REF G\% & VAR A\% & REF T\% & VAR C\% \\
\hline PGM (CLC) & 84.5 & 15.5 & 97 & 3 & 31.5 & 68.5 \\
\hline MiSeq (CLC) & 82 & 18 & 96 & 4 & 12 & 88 \\
\hline PGM (Galaxy) & 84.5 & 15.5 & 97 & 3 & 21.5 & 78.5 \\
\hline MiSeq (Galaxy) & 82 & 18 & 95 & 5 & 11 & 89 \\
\hline PGM (GM-HTS) & 85 & 15.0 & 97 & 3 & 13 & 83 \\
\hline MiSeq (GM-HTS) & 83 & 17.0 & 97 & 3 & 12 & 88 \\
\hline PGM (STRait Razor) & 84 & 16 & 97 & 3 & 12 & 88 \\
\hline MiSeq (STRait Razor) & 83 & 17 & 96 & 4 & 11 & 89 \\
\hline
\end{tabular}

\begin{tabular}{|c|c|c|c|c|c|c|}
\hline \multirow{2}{*}{$C$} & \multicolumn{7}{|c|}{ SRM-HL-60 } \\
\cline { 2 - 7 } & \multicolumn{2}{|c|}{ Position 2445 } & \multicolumn{2}{c|}{ Position 5149 } & \multicolumn{2}{c|}{ Position 12071} \\
\hline Platforms \& Analysis & REF T\% & VAR C\% & REF C\% & VAR T\% & REF T\% & VAR C\% \\
\hline PGM (CLC) & 92.5 & 7.5 & 91.5 & 8.5 & 50 & 50 \\
\hline MiSeq (CLC) & 91 & 9 & 94 & 6 & 50 & 50 \\
\hline PGM (Galaxy) & 92.5 & 7.5 & 91.5 & 8.5 & 49.5 & 50.5 \\
\hline MiSeq (Galaxy) & 91 & 9 & 93 & 7 & 49 & 51 \\
\hline PGM (GM-HTS) & 93 & 7 & 93 & 7 & 54 & 46 \\
\hline MiSeq (GM-HTS) & 93 & 6 & 94 & 6 & 52 & 48 \\
\hline PGM (STRait Razor) & 93 & 7 & 92 & 8 & 50 & 50 \\
MiSeq (STRait Razor) & 92 & 8 & 91 & 9 & 49 & 51 \\
\hline
\end{tabular}

Table 3: Values for all point heteroplasmies in mtDNA SRMs generated on PGM and MiSeq NGS platforms using four different bioinformatics software (CLC bio, Galaxy, GM-HTS, and STRait Razor). (A) SRM-CHR; (B) SRM-9947A; (C) SRM-HL-60. The variant frequencies for PHP in PGM reads depicted in the table are the average of the variant frequency levels found in both PCR 1 and PCR2 reads. The variation observed in the quantitation of mixed bases at 7861 site (SRM-9947A) with the reads generated on the PGM platform is highlighted in bold font. 


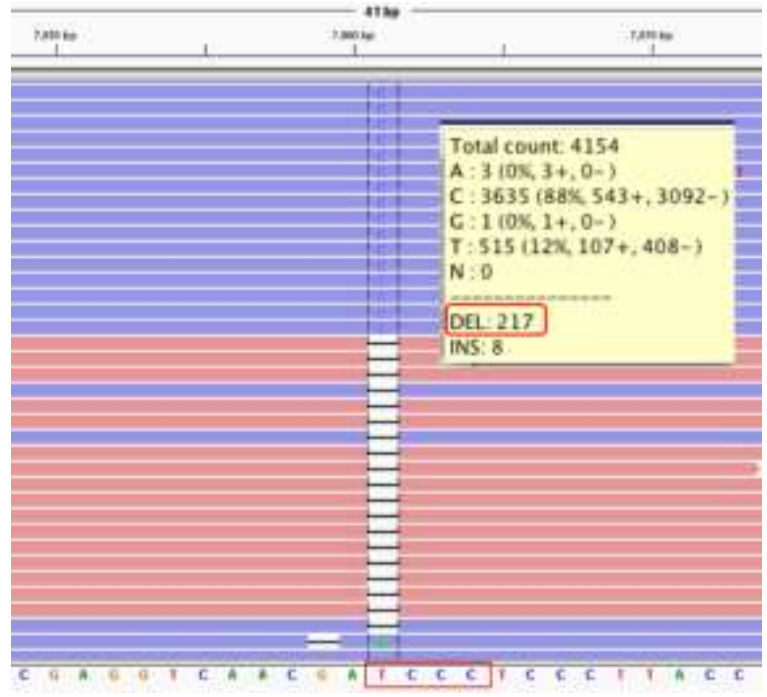

SRM-9947A in IGV before performing any quality-filtering. Sequence pattern of reads containing 7861 position generated on PGM (left panel) and MiSeq (right panel). The 7861 site resides immediately before a homopolymer tract consisting of $>3$ identical bases of Cs (red box). The PGM sequencing reads showed a tendency to be miscalled as seen with the deletion pattern and at the 7861 site (left panel). However, low number of false deletions were detected with the MiSeq data (right panel).

All point heteroplasmies analyzed from the mtDNA SRMs' output data generated on

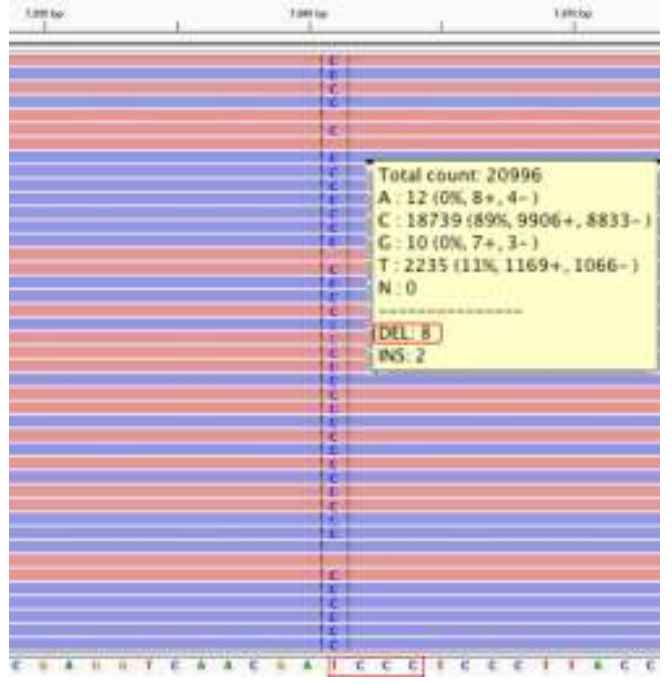

Figure 1. Visualization of BAM files of the both NGS platforms were compared with those previously obtained in Sanger sequencing data. Clear double peaks were observed in all Sanger sequencing reads for PHP $\geq 20 \%$, such as 64 (C/T) (Figure S3) in SRM-CHR and 12,071 (C/T) (data not shown) in SRM-HL60. Sanger sequencing has several limitations due to its restricted sensitivity and its inability to accurately detect and quantitate low-level heteroplasmy. As shown in Figure 2, 1393 heteroplasmy site in SRM-9947A was not always detected by Sanger Sequencing. The mixed bases $(\mathrm{G} / \mathrm{A})$ at 1393 were ambiguous and not reproducible in all reads. Three out of six Sanger reads reveal low level heteroplasmy (Figure 2A). However, the site was detected from the NGS data generated on both PGM and MiSeq, showing an agreement across platforms (Figure 2B). The 3242 site was not reported as a variant from the rCRS with Sanger studies and the 7861 site was reported in the certificate as a homoplasmic variant (7861 C). Upon revisiting the Sanger sequence electropherograms for SRM-9947A (Figure S4), one out of three reads show a mixed base at 3242 (Figure S4) and a mixture of T and C 
bases was indeed present at 7861 (Figure S4), but was not considered due to lack of reproducibility.

A.

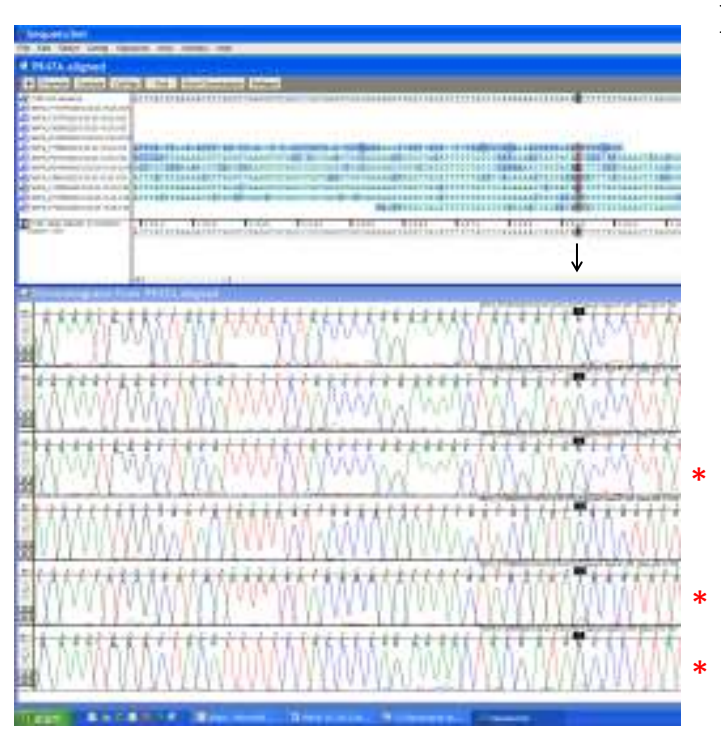

B.

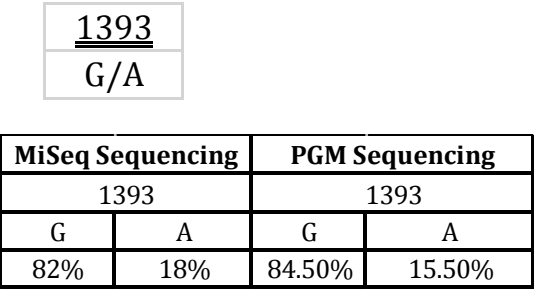

\section{Figure 2. Comparison between data generated with Sanger Sequencing and NGS} platforms.

Panel A. shows the level of heteroplasmy at 1393 in SRM-9947A was not always detectable and reproducible in all Sanger reads. The mixed bases obtained from Sanger Sequencing reads are noted in red asterisk on the side. Only 3 out of six reads show the mixed bases at 1393 position (red asterisk) in SRM-9947A. The 3 other reads showed no evidence of heteroplasmy. Panel B. shows allele frequency observed by both NGS platforms for each base (G or A) at the heteroplasmic position 1393 in SRM-9947A.

In SRM-CHR (Table 1A and S2), an insertion of a C at position 309.1 in HV2 as well as the transition of $\mathrm{T}$ to $\mathrm{C}$ at position 16189 in HV1 region produced two instances of LHP. The insertions of two Cs at 309.1 and 309.2 relative to the reference sequence lead to a length heteroplasmic situation in the HV2 of SRM-9947A (Table 1B and S2). In the SRMHL-60, a C insertion at 315.1 did not cause a length heteroplasmy (Table 1C).

All C-insertions in the HV1 and/or HV2 regions of the SRMs (Table 1) were not 342 properly called according to forensic nomenclature rules [65] using CLC and Galaxy software packages.

In order to resolve, fully capture, and accurately confirm insertions and LHP calls in the homopolymeric regions from the NGS data of the mtDNA SRMs, we used STRait Razor. 
This step further provided information not possible with Sanger Sequencing, which produced uninterpretable sequences downstream of LHP [57].

All C-insertions in each SRM were determined and yielded full concordance with those obtained with Sanger and published in the certificates [14, 16]. However, we were able to further resolve the LHP found in HV1 and HV2 regions with NGS (Table S2). We only examined the sequence calls that were detected in the reads generated from both PGM and MiSeq for concordance. Any pattern that was called only on one platform was discarded (Table S2).

In SRM-CHR, the length types seen with Sanger, an i.e. $\mathrm{C}_{10}$ in $\mathrm{HV} 1$ and $\mathrm{C}_{8}-\mathrm{T}_{-} \mathrm{C}_{6}$ in HV2, were concordant to the ones generated on the NGS platforms. However, we further noticed additional patterns of the Poly-C tract with SRM-CHR NGS data, 4 in the HV1 C-stretch ( $\mathrm{C}_{8}$, $\left.\mathrm{C}_{9}, \mathrm{C}_{11}, \mathrm{C}_{12}\right)$ and 2 in the HV2 region $\left(\mathrm{C}_{7}-\mathrm{T}-\mathrm{C}_{6} ; \mathrm{C}_{9}-\mathrm{T}-\mathrm{C}_{6}\right)$ (Table S2).

Besides the prominent LHP $\left(\mathrm{C}_{9}-\mathrm{T}-\mathrm{C}_{6}\right)$ seen in the HV2 C-stretch of SRM-9947A, NGS revealed another two low LHP levels $\left(\mathrm{C}_{8}-\mathrm{T}-\mathrm{C}_{6} ; \mathrm{C}_{10}-\mathrm{T}-\mathrm{C}_{6}\right)$ (Table S2).

To get an estimate of the frequency of each LHP, we divided the number of reads for a given length by the total number of detected tracts generated by this method (Table S2). Although, length variants sequenced on PGM have same pattern to those generated on MiSeq, we noticed that proportions of LHP within each SRM are different across the platforms and don't agree on reporting the dominant length variant (Table S2). This difference strongly depends on the sequencing technology and chemistry used especially when sequencing along long stretches of homopolymers [51, 63, 64].

The C-insertions in the HV1 and/or HV2 regions were also assessed using GM-HTS. The GM-HTS package has been reported to provide an improved alignment and reporting of length variants [46]. The software eliminates poor alignment by applying a built-in or user-customized motif file to the aligned reads $[46,47]$. All C-insertions in reads generated on MiSeq platform were called properly with the mtDNA typing nomenclature. When analyzing PGM reads, a minimal manual interpretation was required to call the $16193.1 \mathrm{C}$ insertion in SRM-CHR (data not shown).

\section{Quality Control Measures}


In this study low frequency heteroplasmy variants were difficult to detect in Sanger 377 data (Figure S3 and S4) and were only reproducibly seen with the NGS-based studies. 378 However, low-level mixed mtDNA bases detected can be misinterpreted due to the 379 presence of Nuclear mitochondrial Sequences (NumtS), misalignment issues especially around homopolymer regions, sequencing errors, polymorphisms in the primer region, or errors induced during the PCR process that are propagated with each PCR cycle and becomes difficult to distinguish from true low level PHP.

NumtS are DNA tracts located on nuclear chromosomes that are homologous to regions in the mtDNA genome [66]. A combination of several steps aided in limiting NumtS amplification and thoroughly characterizing true low-level PHP. The DNA samples were initially enriched for mtDNA by amplifying the mtDNA genome using long-range PCR primer approach that is designed to avoid nuclear genome amplification [58,67]. The risk of co-amplifying the NumtS was very minimal since none of the primer sets overlap with NumtS sequence tracts [68] that span a near or perfect range to that of the PCR amplicons. Stringent alignment algorithms and parameters were used in both CLC and Galaxy (Figure S1 and S2) that discarded reads that did not map uniquely to the mtGenome. Also, raw sequencing reads from both PGM and MiSeq platforms were mapped in Galaxy against the human genome build hg19 (with Homo sapiens mitochondrion sequence "ChrM" replaced by the rCRS, NC_012920.1) using the BWA-MEM alignment algorithm [42-45]. After selection for the reads that mapped uniquely to the mtGenome, we then filtered out all nonmitochondrial hits, and then assessed point heteroplasmy following the workflow in Figure S2. We did not see any discrepancies between mapping to the hg19 or to rCRS. There was concordance in the calling and the frequency of the low mtDNA heteroplasmic variants.

To control for possible polymerase induced errors, amplification of each SRM 400 material was performed twice before sequencing on the PGM platform and only heteroplasmies reproducible among PCR replicates were considered. Furthermore, a close examination revealed that none of the mtDNA heteroplasmy sites were residing within the primer binding sites ruling out false positive variants due to a PCR primer successfully binding over a variant.

One site, 16183 in SRM-CHR, was flagged as a potential point heteroplasmy "A/C" when both PGM and MiSeq reads were analyzed with the CLC and Galaxy pipelines. The 
16183 position resides immediately before the HV1 C-stretch region "16184-16193". The HV1 C-stretch in SRM-CHR has been shown with Sanger data [14-16] and our NGS study to harbor LHP (insertion variation) (Table 1A and Table S2). To confirm if 16183 is a true heteroplasmy, we further investigated the site using the stringent STRait Razor software tool. Substantial numbers of reads detected by STRait Razor showed either to have insertions or deletions of Cs between 16182-16194 further confirming that this HV1 region exhibits length heteroplasmy. Due to misalignment issues caused by alignment algorithms when dealing with indels, the 16183 has been erroneously called heteroplasmy and was not reported in the list (Table $1 \mathrm{~A})$ as a heteroplasmy site $(\mathrm{A} / \mathrm{C})$ but rather as homoplasmy "C" variant, as it has been detected with Sanger data.

\section{Reproducibility of NGS for mtDNA Heteroplasmy Detection}

In order to account for platform bias [69-71] and provide confidence in our heteroplamsy calls, we compared sequencing data generated on the PGM platform to that generated on the MiSeq. As described above, we reported base calls (Table 1) that we observed to be fully concordant between the two different NGS platforms using all the bioinformatics pipelines including STRait Razor. Analysis of the results from two replicate PCR runs carried out on PGM for each SRM template further revealed the confidence, reproducibility and accuracy of the mtDNA heteroplasmy sites as well as the other variants. These sites were also seen in additional mtDNA SRM NGS data generated using several amplification approaches ("12-amplicon" [72] and "3-amplicon" [73] approaches), library preparation methods, and sequencing platforms (Life Technologies' SOLiD 5500 and HiSeq (Illumina)) (data was not shown). This rules out potential amplification and/or platformspecific bias and strongly suggests that all mixed mtDNA data observed at given nucleotide positions and mentioned in this report are representative of true biological variation in these reference materials.

\section{CONCLUSION}

In this study, sequencing the mtGenome of the three mtDNA SRM templates using PGM and MiSeq NGS chemistries allowed us to simultaneously (i) characterize the complete nucleotide sequence for the existing mtDNA SRMs so that our data can provide a 
positive control and Certified Reference Materials for laboratories wishing to implement and validate any NGS technology, (ii) uncover and annotate additional low level heteroplasmic variants in SRM-9947A and SRM-HL-60, and (iii) quantify the mtDNA heteroplasmy levels at each position using multiple software packages.

Overall, the results of this study provide concordant mtDNA base calls with Sanger data. The majority of the NGS-Sanger discrepancies are related to the low level heteroplasmies and increased sensitivity of the new technology. Evaluation of raw FASTQ reads with multiple software tools help in distinguishing true heteroplasmy calls, interpreting erroneous ones caused by alignment artifacts especially around the indel regions, and investigating discrepancies in variant frequencies as seen with 7861 (SRM9947A). The PHP sites detected on both the MiSeq and PGM platforms, in both PCR replicates, and across the different bioinformatics pipelines will be added with their quantitative values as information values in the updated SRM certificates.

\section{Conflict of interest}

The authors declare no conflict of interest.

\section{NIST funding sources and disclaimers}

This work was funded in part by the Federal Bureau of Investigation (FBI) interagency agreement DJF-13-0100-PR-0000080: "DNA as a Biometric". Points of view in this document are those of the authors and do not necessarily represent the official position or policies of the U.S. Department of Justice or the National Institute of Standards and Technology. Certain commercial equipment, instruments, and materials are identified in order to specify experimental procedures as completely as possible. In no case does such identification imply a recommendation or endorsement by the National Institute of Standards and Technology, nor does it imply that any of the materials, instruments, or equipment identified are necessarily the best available for the purpose.

\section{Acknowledgements}

The authors would like to thank Drs. Katherine B. Gettings, Michael D. Coble, and Justin M. Zook at NIST as well as Dr. Toni M. Diegoli at the Defense Forensic Science Center for their 
470 helpful discussions regarding this manuscript. Also, the authors would like to thank Dr.

471 Teresa Snyder-Leiby and Kayla Hendricks from SoftGenetics for their assistance with the 472 GeneMarker ${ }^{\circledR}$ HTS software. 


\section{References:}

[1] M.M. Holland, D.L. Fisher, L.G. Mitchell, W.C. Rodriquez, J.J. Canik, C.R. Merril, V.W. Weedn, Mitochondrial DNA sequence analysis of human skeletal remains: identification of remains from the Vietnam War, Journal of forensic sciences 38(3) (1993) 542-53. [2] M.M. Holland, D.L. Fisher, R.K. Roby, J. Ruderman, C. Bryson, V.W. Weedn, Mitochondrial DNA sequence analysis of human remains, Crime Laboratory Digest 22(4) (1995) 109-115. [3] M.D. Coble, O.M. Loreille, M.J. Wadhams, S.M. Edson, K. Maynard, C.E. Meyer, H. Niederstatter, C. Berger, B. Berger, A.B. Falsetti, P. Gill, W. Parson, L.N. Finelli, Mystery solved: the identification of the two missing Romanov children using DNA analysis, PloS one 4(3) (2009) e4838.

[4] R. Higuchi, C.H. von Beroldingen, G.F. Sensabaugh, H.A. Erlich, DNA typing from single hairs, Nature 332(6164) (1988) 543-6.

[5] T.D. Anderson, J.P. Ross, R.K. Roby, D.A. Lee, M.M. Holland, A validation study for the extraction and analysis of DNA from human nail material and its application to forensic casework, Journal of forensic sciences 44(5) (1999) 1053-6.

488 [6] C. Ginther, L. Issel-Tarver, M.C. King, Identifying individuals by sequencing mitochondrial DNA from 489 teeth, Nature genetics 2(2) (1992) 135-8.

490 [7] A. Sajantila, A.H. Salem, P. Savolainen, K. Bauer, C. Gierig, S. Paabo, Paternal and maternal DNA lineages reveal a bottleneck in the founding of the Finnish population, Proceedings of the National Academy of Sciences of the United States of America 93(21) (1996) 12035-9.

[8] L. Kang, H.X. Zheng, F. Chen, S. Yan, K. Liu, Z. Qin, L. Liu, Z. Zhao, L. Li, X. Wang, Y. He, L. Jin, mtDNA lineage expansions in Sherpa population suggest adaptive evolution in Tibetan highlands, Molecular biology and evolution 30(12) (2013) 2579-87.

[9] A.W. El-Hattab, F. Scaglia, Mitochondrial DNA depletion syndromes: review and updates of genetic basis, manifestations, and therapeutic options, Neurotherapeutics : the journal of the American Society for Experimental NeuroTherapeutics 10(2) (2013) 186-98.

[10] T. Silkjaer, J.M. Norgaard, A. Aggerholm, L.H. Ebbesen, E. Kjeldsen, P. Hokland, C.G. Nyvold, Characterization and prognostic significance of mitochondrial DNA variations in acute myeloid leukemia, European journal of haematology 90(5) (2013) 385-96.

[11] K. Uzawa, T. Baba, F. Uchida, M. Yamatoji, A. Kasamatsu, Y. Sakamoto, K. Ogawara, M. Shiiba, H. Bukawa, H. Tanzawa, Circulating tumor-derived mutant mitochondrial DNA: a predictive biomarker of clinical prognosis in human squamous cell carcinoma, Oncotarget 3(7) (2012) 670-7.

[12] J. Nunnari, A. Suomalainen, Mitochondria: in sickness and in health, Cell 148(6) (2012) 1145-59.

[13] H.R. Griffin, A. Pyle, E.L. Blakely, C.L. Alston, J. Duff, G. Hudson, R. Horvath, I.J. Wilson, M. Santibanez-Koref, R.W. Taylor, P.F. Chinnery, Accurate mitochondrial DNA sequencing using off-target reads provides a single test to identify pathogenic point mutations, Genetics in medicine : official journal of the American College of Medical Genetics 16(12) (2014) 962-71.

[14] B.C. Levin, K.A. Holland, D.K. Hancock, M. Coble, T.J. Parsons, L.J. Kienker, D.W. Williams, M. Jones, K.L. Richie, Comparison of the complete mtDNA genome sequences of human cell lines--HL-60 and GM10742A--from individuals with pro-myelocytic leukemia and leber hereditary optic neuropathy, respectively, and the inclusion of HL-60 in the NIST human mitochondrial DNA standard reference material--SRM 2392-I, Mitochondrion 2(6) (2003) 387-400.

[15] B.C. Levin, H. Cheng, D.J. Reeder, A human mitochondrial DNA standard reference material for quality control in forensic identification, medical diagnosis, and mutation detection, Genomics 55(2) (1999) 135-46. 
[16] B.C. Levin, H. Cheng, M.C. Kline, J.W. Redman, K.L. Richie, A review of the DNA standard reference materials developed by the National Institute of Standards and Technology, Fresenius' journal of analytical chemistry 370(2-3) (2001) 213-9.

[17] M.R. Wilson, J.A. DiZinno, D. Polanskey, J. Replogle, B. Budowle, Validation of mitochondrial DNA sequencing for forensic casework analysis, International journal of legal medicine 108(2) (1995) 68-74. [18] F. Sanger, S. Nicklen, A.R. Coulson, DNA sequencing with chain-terminating inhibitors, Proceedings of the National Academy of Sciences of the United States of America 74(12) (1977) 5463-7.

[19] M.M. Holland, T.J. Parsons, Mitochondrial DNA Sequence Analysis - Validation and Use for Forensic Casework, Forensic science review 11(1) (1999) 21-50.

[20] W. Parson, A. Dur, EMPOP--a forensic mtDNA database, Forensic science international. Genetics $1(2)(2007) 88-92$.

[21] J.L. King, B.L. LaRue, N.M. Novroski, M. Stoljarova, S.B. Seo, X. Zeng, D.H. Warshauer, C.P. Davis, W. Parson, A. Sajantila, B. Budowle, High-quality and high-throughput massively parallel sequencing of the human mitochondrial genome using the Illumina MiSeq, Forensic science international. Genetics 12 (2014) 128-35.

[22] J.D. Churchill, J. Chang, J. Ge, N. Rajagopalan, S.C. Wootton, C.W. Chang, R. Lagace, W. Liao, J.L. King, B. Budowle, Blind study evaluation illustrates utility of the lon PGM system for use in human identity DNA typing, Croatian medical journal 56(3) (2015) 218-29.

[23] M.D. Coble, R.S. Just, J.E. O'Callaghan, I.H. Letmanyi, C.T. Peterson, J.A. Irwin, T.J. Parsons, Single nucleotide polymorphisms over the entire mtDNA genome that increase the power of forensic testing in Caucasians, International journal of legal medicine 118(3) (2004) 137-46.

[24] T.J. Parsons, M.D. Coble, Increasing the forensic discrimination of mitochondrial DNA testing through analysis of the entire mitochondrial DNA genome, Croatian medical journal 42(3) (2001) 304-9. [25] S. Anderson, A.T. Bankier, B.G. Barrell, M.H. de Bruijn, A.R. Coulson, J. Drouin, I.C. Eperon, D.P. Nierlich, B.A. Roe, F. Sanger, P.H. Schreier, A.J. Smith, R. Staden, I.G. Young, Sequence and organization of the human mitochondrial genome, Nature 290(5806) (1981) 457-65.

[26] M.M. Holland, M.R. McQuillan, K.A. O'Hanlon, Second generation sequencing allows for mtDNA mixture deconvolution and high resolution detection of heteroplasmy, Croatian medical journal 52(3) (2011) 299-313.

[27] M. Mikkelsen, E. Rockenbauer, A. Wächter, L. Fendt, B. Zimmermann, W. Parson, S.A. Nielsen, T. Gilbert, E. Willerslev, N. Morling, Application of full mitochondrial genome sequencing using 454 GS FLX pyrosequencing, Forensic Science International: Genetics Supplement Series 2(1) 518-519.

[28] O. Loreille, H. Koshinsky, V.Y. Fofanov, J.A. Irwin, Application of next generation sequencing technologies to the identification of highly degraded unknown soldiers\&\#x2019; remains, Forensic Science International: Genetics Supplement Series 3(1) e540-e541.

[29] H. Goto, B. Dickins, E. Afgan, I.M. Paul, J. Taylor, K.D. Makova, A. Nekrutenko, Dynamics of mitochondrial heteroplasmy in three families investigated via a repeatable re-sequencing study, Genome biology 12(6) (2011) R59.

[30] B. Rebolledo-Jaramillo, M.S. Su, N. Stoler, J.A. McElhoe, B. Dickins, D. Blankenberg, T.S. Korneliussen, F. Chiaromonte, R. Nielsen, M.M. Holland, I.M. Paul, A. Nekrutenko, K.D. Makova, Maternal age effect and severe germ-line bottleneck in the inheritance of human mitochondrial DNA, Proceedings of the National Academy of Sciences of the United States of America 111(43) (2014) 154749. [31] M.A. Quail, M. Smith, P. Coupland, T.D. Otto, S.R. Harris, T.R. Connor, A. Bertoni, H.P. Swerdlow, Y. $\mathrm{Gu}, \mathrm{A}$ tale of three next generation sequencing platforms: comparison of lon Torrent, Pacific Biosciences and Illumina MiSeq sequencers, BMC genomics 13 (2012) 341. 
608

609

610

[32] S. Junemann, F.J. Sedlazeck, K. Prior, A. Albersmeier, U. John, J. Kalinowski, A. Mellmann, A. Goesmann, A. von Haeseler, J. Stoye, D. Harmsen, Updating benchtop sequencing performance comparison, Nature biotechnology 31(4) (2013) 294-6.

[33] B.J. Bintz, G.B. Dixon, M.R. Wilson, Simultaneous detection of human mitochondrial DNA and nuclear-inserted mitochondrial-origin sequences (NumtS) using forensic mtDNA amplification strategies and pyrosequencing technology, Journal of forensic sciences 59(4) (2014) 1064-73.

[34] M. Mikkelsen, R. Frank-Hansen, A.J. Hansen, N. Morling, Massively parallel pyrosequencing of the mitochondrial genome with the 454 methodology in forensic genetics, Forensic science international. Genetics 12 (2014) 30-7.

[35] J.A. McElhoe, M.M. Holland, K.D. Makova, M.S. Su, I.M. Paul, C.H. Baker, S.A. Faith, B. Young, Development and assessment of an optimized next-generation DNA sequencing approach for the mtgenome using the Illumina MiSeq, Forensic science international. Genetics 13 (2014) 20-9.

[36] Human mtDNA Genome. <http://support.illumina.com/content/dam/illuminasupport/documents/documentation/chemistry documentation/samplepreps legacy/human-mtdnagenome-guide-15037958-01.pdf $>$ ).

[37] B.J.B. H. Stawski, E. S. Burnside, and M. Wilson, Preparing Whole Genome Human Mitochondrial DNA Libraries for Next Generation Sequencing (NGS) Using Illumina Nextera XT, Poster presentation at the 65th Annual American Academy of Forensic Sciences Conference, Washington, D.C., 2013.

[38] Ion Xpress ${ }^{\mathrm{TM}}$ Plus and Ion Plus Library Preparation for the AB Library Builder ${ }^{\mathrm{TM}}$ System.

<https://tools.thermofisher.com/content/sfs/manuals/MAN0006946 IonXpressLibraryPrepforABLB UG $. \mathrm{pdf}>)$.

[39] Ion Library TaqMan ${ }^{\circledR}$ Quantitation Kit USER GUIDE.

$<$ https://tools.thermofisher.com/content/sfs/manuals/MAN0015802 lonLibrary Taqman Quantitation Kit UG.pdf $>$ ).

[40] Ion PGM ${ }^{\text {тм }} \mathrm{Hi}-\mathrm{Q}^{\text {тM }}$ Chef Kit USER GUIDE.

<https://tools.thermofisher.com/content/sfs/manuals/MAN0010919 lonHiQ Chef UG.pdf>).

[41] CLC bio Genomics Workbench version 9.0. $<$ http://resources.qiagenbioinformatics.com/manuals/clcgenomicsworkbench/702/User Manual.pdf>). [42] D. Blankenberg, J. Taylor, I. Schenck, J. He, Y. Zhang, M. Ghent, N. Veeraraghavan, I. Albert, W. Miller, K.D. Makova, R.C. Hardison, A. Nekrutenko, A framework for collaborative analysis of ENCODE data: making large-scale analyses biologist-friendly, Genome research 17(6) (2007) 960-4.

[43] J. Goecks, A. Nekrutenko, J. Taylor, Galaxy: a comprehensive approach for supporting accessible, reproducible, and transparent computational research in the life sciences, Genome biology 11(8) (2010) R86.

[44] Public Galaxy Instance. <http://usegalaxy.org>).

[45] Galaxy page describing analysis of mitochondrial heteroplasmy. <http://usegalaxy.org/heteroplasmy>).

[46] M.M. Holland, E.D. Pack, J.A. McElhoe, Evaluation of GeneMarker(R) HTS for improved alignment of mtDNA MPS data, haplotype determination, and heteroplasmy assessment, Forensic science international. Genetics 28 (2017) 90-98.

[47] GeneMarker ${ }^{\circledR}$ HTS Mitochondrial NGS Analysis Software. <http://www.softgenetics.com/PDF/GeneMarkerHTS UsersManual.pdf>). [48] R.M. Andrews, I. Kubacka, P.F. Chinnery, R.N. Lightowlers, D.M. Turnbull, N. Howell, Reanalysis and revision of the Cambridge reference sequence for human mitochondrial DNA, Nature genetics 23(2) (1999) 147.

[49] D.H. Warshauer, J.L. King, B. Budowle, STRait Razor v2.0: the improved STR Allele Identification Tool--Razor, Forensic science international. Genetics 14 (2015) 182-6. 
[50] D.H. Warshauer, D. Lin, K. Hari, R. Jain, C. Davis, B. Larue, J.L. King, B. Budowle, STRait Razor: a length-based forensic STR allele-calling tool for use with second generation sequencing data, Forensic science international. Genetics 7(4) (2013) 409-17. [51] X. Li, A.J. Buckton, S.L. Wilkinson, S. John, R. Walsh, T. Novotny, I. Valaskova, M. Gupta, L. Game, P.J. Barton, S.A. Cook, J.S. Ware, Towards clinical molecular diagnosis of inherited cardiac conditions: a comparison of bench-top genome DNA sequencers, PloS one 8(7) (2013) e67744.

[52] H. Li, N. Homer, A survey of sequence alignment algorithms for next-generation sequencing, Briefings in bioinformatics 11(5) (2010) 473-83.

[53] J. Shendure, H. Ji, Next-generation DNA sequencing, Nature biotechnology 26(10) (2008) 1135-45. [54] R.N. Lightowlers, P.F. Chinnery, D.M. Turnbull, N. Howell, Mammalian mitochondrial genetics: heredity, heteroplasmy and disease, Trends in genetics : TIG 13(11) (1997) 450-5.

[55] A. Salas, M.V. Lareu, A. Carracedo, Heteroplasmy in mtDNA and the weight of evidence in forensic mtDNA analysis: a case report, International journal of legal medicine 114(3) (2001) 186-90.

[56] B. Budowle, M.W. Allard, M.R. Wilson, R. Chakraborty, Forensics and mitochondrial DNA: applications, debates, and foundations, Annual review of genomics and human genetics 4 (2003) 11941.

[57] W. Parson, L. Gusmao, D.R. Hares, J.A. Irwin, W.R. Mayr, N. Morling, E. Pokorak, M. Prinz, A. Salas, P.M. Schneider, T.J. Parsons, DNA Commission of the International Society for Forensic Genetics: revised and extended guidelines for mitochondrial DNA typing, Forensic science international. Genetics 13 (2014) 134-42.

[58] R.S. Just, J.A. Irwin, W. Parson, Mitochondrial DNA heteroplasmy in the emerging field of massively parallel sequencing, Forensic science international. Genetics 18 (2015) 131-9.

[59] C. Berger, P. Hatzer-Grubwieser, C. Hohoff, W. Parson, Evaluating sequence-derived mtDNA length heteroplasmy by amplicon size analysis, Forensic science international. Genetics 5(2) (2011) 142-5. [60] J.A. Irwin, J.L. Saunier, H. Niederstatter, K.M. Strouss, K.A. Sturk, T.M. Diegoli, A. Brandstatter, W. Parson, T.J. Parsons, Investigation of heteroplasmy in the human mitochondrial DNA control region: a synthesis of observations from more than 5000 global population samples, Journal of molecular evolution 68(5) (2009) 516-27.

[61] K.M. Kiesler, P.M. Vallone, Characterization of NIST standard reference materials by next generation sequencing, Forensic Science International: Genetics Supplement Series 4(1) e97-e98.

[62] J.T. Robinson, H. Thorvaldsdottir, W. Winckler, M. Guttman, E.S. Lander, G. Getz, J.P. Mesirov, Integrative genomics viewer, Nature biotechnology 29(1) (2011) 24-6.

[63] B. Merriman, J.M. Rothberg, Progress in ion torrent semiconductor chip based sequencing, Electrophoresis 33(23) (2012) 3397-417.

[64] N.J. Loman, R.V. Misra, T.J. Dallman, C. Constantinidou, S.E. Gharbia, J. Wain, M.J. Pallen, Performance comparison of benchtop high-throughput sequencing platforms, Nature biotechnology 30(5) (2012) 434-9. [65] SWGDAM Mitochondrial DNA Nomenclature Examples. <http://media.wix.com/ugd/4344b0 2044739c57574dbea97f2f85b6f73c9d.pdf>).

[66] E. Hazkani-Covo, R.M. Zeller, W. Martin, Molecular poltergeists: mitochondrial DNA copies (numts) in sequenced nuclear genomes, PLoS genetics 6(2) (2010) e1000834.

[67] M. Li, R. Schroeder, A. Ko, M. Stoneking, Fidelity of capture-enrichment for mtDNA genome sequencing: influence of NUMTs, Nucleic acids research 40(18) (2012) e137.

[68] F.M. Calabrese, D. Simone, M. Attimonelli, Primates and mouse NumtS in the UCSC Genome Browser, BMC bioinformatics 13 Suppl 4 (2012) S15.

[69] S. Seneca, K. Vancampenhout, R. Van Coster, J. Smet, W. Lissens, A. Vanlander, B. De Paepe, A. Jonckheere, K. Stouffs, L. De Meirleir, Analysis of the whole mitochondrial genome: translation of the 
658 Ion Torrent Personal Genome Machine system to the diagnostic bench?, European journal of human 659 genetics : EJHG 23(1) (2015) 41-8.

660 [70] M. Li, A. Schonberg, M. Schaefer, R. Schroeder, I. Nasidze, M. Stoneking, Detecting heteroplasmy 661 from high-throughput sequencing of complete human mitochondrial DNA genomes, American journal of 662 human genetics 87(2) (2010) 237-49.

663 [71] X.V. Wang, N. Blades, J. Ding, R. Sultana, G. Parmigiani, Estimation of sequencing error rates in short 664 reads, BMC bioinformatics 13 (2012) 185.

665 [72] J.A. Irwin, J.L. Saunier, K.M. Strouss, K.A. Sturk, T.M. Diegoli, R.S. Just, M.D. Coble, W. Parson, T.J. 666 Parsons, Development and expansion of high-quality control region databases to improve forensic 667 mtDNA evidence interpretation, Forensic science international. Genetics 1(2) (2007) 154-7.

668 [73] A. Maitra, Y. Cohen, S.E. Gillespie, E. Mambo, N. Fukushima, M.O. Hoque, N. Shah, M. Goggins, J. 669 Califano, D. Sidransky, A. Chakravarti, The Human MitoChip: a high-throughput sequencing microarray 670 for mitochondrial mutation detection, Genome research 14(5) (2004) 812-9.

671 